\title{
COMMENT
}

\section{Choosing and using multiple traits in functional diversity research}

\section{Introduction}

Species are different, but they are not equally different. Yet many indices of biodiversity assume species vary to identical degrees (Petchey et al. 2004). This notion does not meet with intuition: some species vary greatly in terms of their morphology, behaviour and ecology, while others vary only a little. One way to reconcile the dissimilarity between species is by collecting information on their functional traits (FTs), descriptors of how organisms interact with their environment and each other. Functional diversity (FD) is the total variation in one or more FTs across all species within a community, and provides a powerful complement to species diversity (Faith 1996). There are several challenges facing the application of FD to conservation science, including lack of rigorous trait data for many organisms, and sparse details on how to select available traits to generate meaningful inferences for the various summary metrics of FD. In this Comment, we provide a brief discussion on choosing and using FTs, and provide recommendations for best practice (Fig. 1). Ultimately, we ask researchers to consider using a variety of traits when hypotheses are multifaceted or could potentially evolve, and at the same time to think critically about trait selection to avoid redundant information.

FTs have been used to explore a wide variety of phenomena, including macroecological patterns (Devictor et al. 2010), mechanisms of community assembly (HilleRisLambers $\mathrm{et} \mathrm{al}$. 2012), maintenance of ecosystem functioning (Hooper et al. 2005), and as indicators for conservation (Stuart-Smith et al. 2013). In many cases, the insight gained through the use of FTs would be impossible or limited with traditional speciesbased approaches. These varied applications, however, mean the definition of FTs has become conflated and increasingly specialized (see supplementary material). Here, we use a broader and more operational definition of FTs as: measurable characteristics of individual organisms relating to morphology, physiology, phenology and behaviour that can be used to explain and compare their responses to the environment and/or their effects on ecosystem functioning (McGill et al. 2006; Díaz et al. 2013).

\section{Traits in a less-than-ideal world}

Empirical estimates of trait importance are lacking for many organisms, systems, and processes. Further, the targets

Supplementary material: available online at http://dx.doi.org/ $10.1017 /$ S0376892914000307 of long-term conservation and management programmes may change over time, and with them the traits that are deemed relevant. Data from multiple traits are more likely to capture the functional responses of communities across the gamut of possible management scenarios (Fig. 1). In contrast, one or two traits might only show a signal under certain circumstances, which may be misleading under new applications. We offer four additional arguments for using multiple FTs when characterizing FD:

(1) When multiple processes are shaping the community under investigation, they may reflect differently in its trait composition. Only by including multiple traits can we achieve a holistic representation of FD. This may be especially relevant when looking at broad spatial and temporal patterns that are influenced by many different factors, including anthropogenic activities.

(2) By extension, different traits may drive different, and potentially unidentified, ecosystem functions (Petchey \& Gaston 2006), and we are often interested in managing diversity to support multiple functions in an inclusive ecosystem framework (de Bello et al. 2010).

(3) Multiple traits allow for post hoc estimation of trait importance (Pillar \& Sosinski 2003; Petchey et al. 2004). Such an exploratory approach may suggest starting points for more focused investigations. It may also identify traits that have no direct link to the observed outcome, and thus might have otherwise been excluded, but which have important indirect consequences that only become evident through such an analysis (Griffin et al. 2009).

(4) Multiple traits may approximate traits that are difficult to measure ('hard traits'), in the sense that differences can be preserved across combinations of other, less intensive traits ('soft traits' sensu Hodgson et al. 1999). This is not to discourage against pursuing difficult but informative traits, but rather acknowledges that the time, money, samples, and effort necessary to obtain such traits may simply not be available (Petchey \& Gaston 2006). These constraints should not preclude an investigation of FD if less rigorous trait data are available, as long as those traits are reasonable proxies.

\section{More is not always better}

A useful FT will vary among species (McGill et al. 2006; Petchey \& Gaston 2006). However, some shared trait values can help quantify the degree of redundancy within assemblages, and may identify vulnerable ecosystems and services (Rosenfeld 2002). Conversely, FTs that strongly 


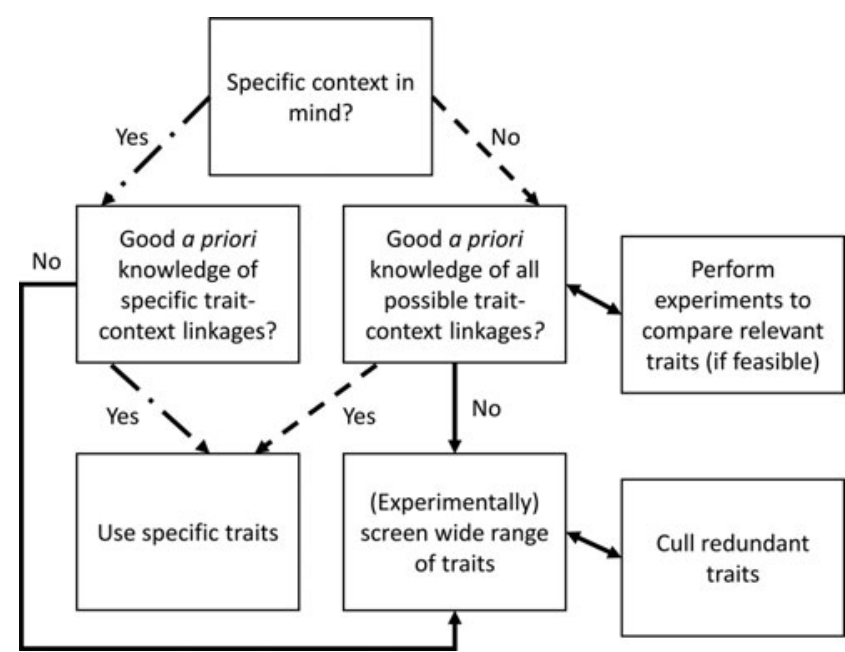

Figure 1 If researchers are quantifying functional diversity with a specific pattern or process in mind, and have a solid understanding of relevant traits, a limited suite of traits with known or theoretically validated explanatory power should be used (dashed-dotted route). In rare cases, even when the researcher is focused on multiple contexts, knowledge of all trait-context linkages may exist (dashed route). More commonly, faced with multiple contexts, a researcher would have incomplete knowledge and should screen a wide range of traits (solid route), and supplement this approach with a trait-culling procedure and/or experiments to increase knowledge of potential trait linkages.

discriminate species can cause indices to mirror species richness, negating a functional approach (Schleuter et al. 2010). Thus, some caution should be exercised when combining multiple traits. Additional traits may also not reveal any new information. Recent investigations using dimensionality reduction and model selection techniques have revealed that between 3-5 traits were necessary to completely characterize plant communities (Laughlin 2014) and species interaction networks (Eklöf et al. 2013). However, these studies favoured plants which, being well-studied and less complex than animals, are more likely to be accurately represented by fewer traits. Finally, FTs should also not be confounded with the pattern or process they are trying to explain. For example, using habitat association as a trait to explain community structure along a spatial gradient would be confounded if habitats were distributed unequally along the same gradient.

In most analyses of FD, multiple traits are collapsed into functional indices, and this process can be equally sensitive to trait choices. For instance, Poos et al. (2009) showed that a dendrogram-based index of FD was sensitive to both the clustering algorithm, and the distance measure used to quantify dissimilarity among species (for example Euclidean or Gower). Pakeman (2014) showed that five common metrics used to quantify $\mathrm{FD}$, namely functional divergence and dispersion, Rao's quadratic entropy, and particularly functional richness and evenness, were sensitive to missing values.

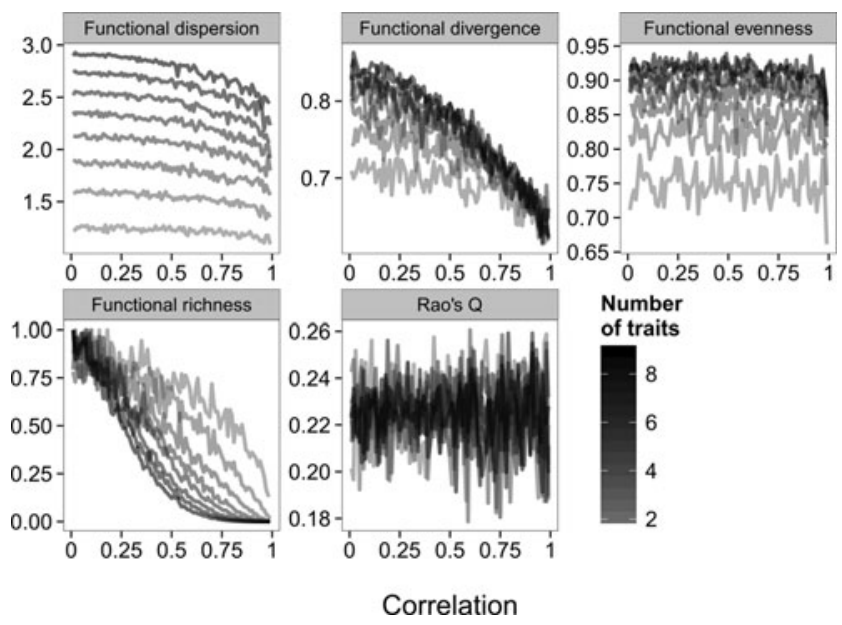

Figure 2 The relationship between five common indices of functional diversity and the degree of correlation among traits using simulated data for 100 species. Shading indicates the number of traits included in the analysis.

There has been limited discussion of how correlations among multiple traits might impact indices of FD (Cornwell et al. 2006, Villéger et al. 2008). To explore this phenomenon, we simulated values for the above indices of FD while varying the degree of correlation, the number of traits, and species richness (see supplementary material). We found that functional dispersion and evenness were largely invariant to correlations among traits, except at very high levels of correlation $(r>0.95$; Fig. 2). Functional richness and divergence decreased substantially with increasing correlations. Correlated traits reduce the absolute volume of multidimensional trait space, which is used in the calculation of these two indices (Cornwell et al. 2006). Generally, declines were exaggerated as more traits were considered (Fig. 2). These trends were robust to changes in species richness (supplementary material Fig. S1). In contrast to Villéger et al. (2008), our simulations suggest that retaining many correlated traits will cause functional richness and divergence to converge on smaller values, based purely on the mathematical properties of the indices.

Several methods have been proposed to deal with the issue of correlations among functions, including dimensionalityreduction techniques like principal coordinates analysis (Villéger et al. 2008; Laliberté \& Legendre 2010). However, these may not preserve actual differences among species (Schleuter et al. 2010), and the components may be uninterpretable within actual biology and ecology. A more sensible and interpretable approach involves culling traits before they are subjected to any kind of calculation, using either a priori knowledge of the system or model selection approaches.

Multivariate indices can also be misleading when there are interactions or many weak relationships among traits. Strong opposing trends among individual traits may result in tradeoffs that cause the multivariate index to show no average trend 
(Spasojevic \& Suding 2012). Similarly, a single strong signal may be swamped in the presence of many weak relationships (Bellwood et al. 2006). It is difficult to anticipate when these kinds of trade-offs or myriad weak relationships might occur. Thus, we recommend calculating both a multivariate index and performing a thorough investigation of each trait individually (for example by using community weighted means; Ricotta \& Moretti 2011).

\section{Moving forward}

There are still many frontiers to explore within the context of functional traits. FTs of individuals may vary within a species, sometimes more than among species. New methods allow integration of this variability at the individual level into calculations of FD (de Bello et al. 2011). Many FTs, particularly those obtained from existing data, represent average values taken across the full range of conditions under which species occur. Such values may not be representative for smaller scale investigations, or account for altered phenotypes driven by global change. Phylogenetic diversity has been proposed as a holistic proxy for functional differences. As with FD, phylogenetic diversity may be sensitive to the genes used and methodological choices during tree construction, which can influence tree topology and resulting inferences (Tucker \& Cadotte 2013). There is limited mechanistic understanding of how variation at the genetic level translates to natural patterns and processes. FTs rectify this issue by drawing on observable phenotypes and interactions, and thus provide insight into the potential ecological mechanisms at work. Here, we advise researchers to carefully consider how trait and methodological choices might affect their research goals. We recommend a multi-trait approach in cases where hypotheses are not absolutely clear, coupled with careful vetting of potentially redundant information.

\section{Acknowledgements}

We thank three anonymous reviewers for their comments on an earlier draft. Jonathan Lefcheck was supported by the VIMS Maury Fellowship. Vinicius Bastazini was funded by CAPES-Brazil. John Griffin was funded by the Climate Change Consortium of Wales $(\mathrm{C} 3 \mathrm{~W})$. This paper is Contribution No. 3392 of the Virginia Institute of Marine Science, College of William \& Mary.

\section{Supplementary materials}

We provide further discussion of the definition of functional traits, and methods and $\mathrm{R}$ code for simulations. To view supplementary material for this article, please visit http://dx.doi.org/10.1017/S0376892914000307

\section{References}

Bellwood, D. R., Wainwright, P. C., Fulton, C. J. \& Hoey, A. S. (2006) Functional versatility supports coral reef biodiversity. Proceedings of the Royal Society B 273: 101-107.
Cornwell, W. K., Schwilk, D. W. \& Ackerly, D. D. (2006) A traitbased test for habitat filtering: convex hull volume. Ecology 87: $1465-1471$.

De Bello, F., Lavorel, S., Díaz, S., Harrington, R., Cornelissen, J. H. C., Bardgett, R. D., Berg, M. P., Cipriotti, P., Feld, C. K., Hering, D., Martins da Silva, P., Potts, S. G., Sandin, L., Sousa, J. P., Storkey, J., Wardle, D. A. \& Harrison, P. A. (2010) Towards an assessment of multiple ecosystem processes and services via functional traits. Biodiversity and Conservation 19: 2873-2893.

De Bello, F., Lavorel, S., Albert, C. H., Thuiller, W., Grigulis, K., Dolezal, J., Janeček, Š. \& Lepš, J. (2011) Quantifying the relevance of intraspecific trait variability for functional diversity. Methods in Ecology and Evolution 2: 163-174.

Devictor, V., Mouillot, D., Meynard, C., Jiguet, F., Thuiller, W. \& Mouquet, N. (2010) Spatial mismatch and congruence between taxonomic, phylogenetic and functional diversity: the need for integrative conservation strategies in a changing world. Ecology Letters 13: 1030-1040.

Díaz, S., Purvis, A., Cornelissen, J. H. C., Mace, G. M., Donoghue, M. J., Ewers, R. M., Jordano, P. \& Pearse, W. D. (2013) Functional traits, the phylogeny of function, and ecosystem service vulnerability. Ecology and Evolution 3: 2958-2975.

Eklöf, A., Jacob, U., Kopp, J., Bosch, J., Castro-Urgal, R., Chacoff, N. P., Dalsgaard, B., de Sassi, C., Galetti, M., Guimarães, P. R., Lomáscolo, S. B., Martín González, A. M., Pizo, M. A., Rader, R., Rodrigo, A., Tylianakis, J. M., Vázquez, D. P. \& Allesina, S. (2013) The dimensionality of ecological networks. Ecology Letters 16: $577-583$

Faith, D. P. (1996) Conservation priorities and phylogenetic pattern. Conservation Biology 10: 1286-1289.

Griffin, J. N., Jenkins, S. R., Gamfeldt, L., Jones, D., Hawkins, S. J. \& Thompson, R. C. (2009) Spatial heterogeneity increases the importance of species richness for an ecosystem process. Oikos 118: $1335-1342$.

HilleRisLambers, J., Adler, P. B., Harpole, W. S., Levine, J. M. \& Mayfield, M. M. (2012) Rethinking community assembly through the lens of coexistence theory. Annual Reviem of Ecology, Evolution, and Systematics 43: 227-248.

Hodgson, J. G., Wilson, P. J., Hunt, R., Grime, J. P. \& Thompson, K. (1999) Allocating CSR plant functional types: a soft approach to a hard problem. Oikos 85: 282-294.

Hooper, D. U., Chapin III, F. S., Ewel, J. J., Hector, A., Inchausti, P., Lavorel, S., Lawton, J. H., Lodge, D. M., Loreau, M., Naeem, S., Schmid, B., Setälä, H., Symstad, A. J., Vandermeer, J. \& Wardle, D. A. (2005) Effects of biodiversity on ecosystem functioning: a consensus of current knowledge. Ecological Monographs 75: 3-35.

Laliberté, E. \& Legendre, P. (2010) A distance-based framework for measuring functional diversity from multiple traits. Ecology 91: 299-305.

Laughlin, D. C. (2014) The intrinsic dimensionality of plant traits and its relevance to community assembly. Fournal of Ecology 102 186-193.

McGill, B. J., Enquist, B. J., Weiher, E. \& Westoby, M. (2006) Rebuilding community ecology from functional traits. Trends in Ecology and Evolution 21: 178-185.

Pakeman, R. J. (2014) Functional trait metrics are sensitive to the completeness of the species' trait data? Methods in Ecology and Evolution 5: 9-15.

Petchey, O. L. \& Gaston, K. J. (2006) Functional diversity: back to basics and looking forward. Ecology Letters 9: 741-758. 
Petchey, O. L., Hector, A. \& Gaston, K. J. (2004) How do different measures of functional diversity perform? Ecology 85: 847-857.

Pillar, V. D. \& Sosinski Jr, E. E. (2003) An improved method for searching plant functional types by numerical analysis. Fournal of Vegetation Science 14: 323-332.

Poos, M. S., Walker, S. C. \& Jackson, D. A. (2009) Functionaldiversity indices can be driven by methodological choices and species richness. Ecology 90: 341-347.

Ricotta, C. \& Moretti, M. (2011) CWM and Rao's quadratic diversity: a unified framework for functional ecology. Oecologia 167: 181188.

Rosenfeld, J. S. (2002) Functional redundancy in ecology and conservation. Oikos 98: 156-162.

Schleuter, D., Daufresne, M., Massol, F. \& Argillier, C. (2010) A user's guide to functional diversity indices. Ecological Monographs 80: 469-484.

Spasojevic, M. J. \& Suding, K. N. (2012) Inferring community assembly mechanisms from functional diversity patterns: the importance of multiple assembly processes. Fournal of Ecology 100: 652-661.

Stuart-Smith, R. D., Bates, A. E., Lefcheck, J. S., Duffy, J. E., Baker, S. C., Thomson, R. J., Stuart-Smith, J. F., Hill, N. a.,
Kininmonth, S. J., Airoldi, L., Becerro, M. A., Campbell, S. J., Dawson, T. P., Navarrete, S. A., Soler, G. A., Strain, E. M. A., Willis, T. J. \& Edgar, G. J. (2013) Integrating abundance and functional traits reveals new global hotspots of fish diversity. Nature 501: 539-542.

Tucker, C. M. \& Cadotte, M. W. (2013) Unifying measures of biodiversity: understanding when richness and phylogenetic diversity should be congruent. Diversity and Distributions 19: 845854.

Villéger, S., Mason, N. W. H. \& Mouillot, D. (2008) New multidimensional functional diversity indices for a multifaceted framework in functional ecology. Ecology 89: 2290-2301.

JONATHAN S. LEFCHECK ${ }^{1 *}$, VINICIUS A. G. BASTAZINI ${ }^{2}$ AND JOHN N. GRIFFIN ${ }^{3}$

${ }^{1}$ Virginia Institute of Marine Science, College of William E Mary, PO Box 1346, Gloucester Point, Virginia 23062-1346, USA, ${ }^{2} P P G-$ Ecologia, UFRGS, Av. Bento Gonçalves 9500, CEP 91501-970, Porto Alegre, Brazil and ${ }^{3}$ Department of Biosciences, Wallace Building, Smansea University, Smansea, SA2 8PP, UK

*Correspondence: Jonathan S. Lefcheck Tel: + 18046847150 e-mail: jslefche@vims.edu 